\title{
Variáveis associadas à mortalidade materna e infantil: uma revisão integrativa
}

\author{
Variables associated with maternal and child mortality: an integrative review \\ Ingra Pereira Monti Martins a \\ Orcid: https://orcid.org/0000-0002-5824-9461 \\ Cristiane Yumi Nakamura ${ }^{b}$ \\ Orcid: https://orcid.org/0000-0003-3064-3085 \\ Deborah Ribeiro Carvalho ${ }^{\mathrm{c}}$ \\ Orcid: https://orcid.org/0000-0002-9735-650X
}

\begin{abstract}
Resumo
Introdução: A mortalidade materna e infantil reflete a situação sanitária e a qualidade da assistência à saúde. As taxas de mortalidade no Brasil apresentam diferenças entre as regiões geográficas e são superiores às de outros países em desenvolvimento. Objetivo: Compreender variáveis associadas à mortalidade materna e infantil. Materiais e Métodos: Revisão integrativa, consultadas as bases Literatura Latino-Americana e do Caribe em Ciências da Saúde, Medical Literature Analysis and Retrieval System Online e Banco de Dados em Enfermagem. Considerados artigos publicados entre 1999-2018, cenário Brasil e idioma português, selecionados por dois pesquisadores calibrados com nível de concordância Kappa substancial. As variáveis associadas significativamente às mortalidades foram aproximadas às camadas do modelo dos Determinantes Sociais de Saúde. Resultados: Identificaram-se 522 artigos e selecionados 74. Por camada, destacaram-se as variáveis: características individuais - peso ao nascer e idade materna; estilo de vida - tabagismo na gestação; influências sociais estado civil materno; condições de vida e trabalho - assistência pré-natal e escolaridade materna; condições socioeconômicas e ambientais gerais - saneamento básico e renda. Conclusões: As variáveis mais encontradas foram peso ao nascer e idade materna, aproximadas à camada individual, de baixa governabilidade direta do gestor. Para mudanças nessa camada, as intervenções devem influenciar nas variáveis das camadas mais externas, das quais destacaram-se assistência pré-natal, escolaridade materna, renda e saneamento básico. Reforça-se a responsabilidade direta dos gestores na redução da mortalidade materna e infantil, por meio de ações que visem reduzir as iniquidades em saúde.
\end{abstract}

Palavras-chave: mortalidade infantil; mortalidade materna; determinantes sociais da saúde.

\footnotetext{
Abstract

Introduction: Maternal and child mortality reflect the local health situation and the quality health care. Mortality rates in Brazil differ between geographic regions and are higher than in others developing countries. Objectives: To comprehend variables associated with maternal and child mortality. Materials and Methods: Integrative review, researching Latin American and Caribbean Health Science Literature, Medical Analysis and Retrieval System Online and Nursing Database, considering only articles published between 1999 and 2018, Brazil scenario and Portuguese language, selected by two researchers calibrated with a substantial Kappa level of agreement. Variables significantly associated with mortality were extracted from articles and approximated to the layers of the Social Determinants of Health. Results: Five hundred and twenty-two articles were identified and 74 were selected. By layer, the following variables

${ }^{a}$ Graduanda em Medicina pela Pontifícia Universidade Católica do Paraná, Brasil. E-mail: ingrapm@hotmail.com

${ }^{\text {b }}$ Mestranda Programa de Pós-graduação em Tecnologia em Saúde Pontifícia, Universidade Católica do Paraná, Brasil. Email: cristy_nakamura@hotmail.com

c Programa de Pós-graduação em Tecnologia em Saúde Pontifícia, Universidade Católica do Paraná, Brasil. E-mail: ribeiro.carvalho@pucpr.br
} 
were highlighted: individual characteristics - birth weight and maternal age; lifestyle - smoking in pregnancy; social influences - maternal marital status; living and working conditions prenatal care and maternal education; general socioeconomic and environmental conditions basic sanitation and rent. Conclusions: The most found variables were birth weight and maternal age, close to the individual layer, with low direct governance of the manager. For changes in this layer, interventions must be influence the variables of the most external layers, of which prenatal care, maternal education, income, and basic sanitation stood out. The direct responsibility of managers in reducing maternal and child mortality is reinforced trough actions aimed at reducing health inequities.

Keywords: infant mortality; maternal mortality; social determinants of health.

\section{Introdução}

A Taxa de Mortalidade Infantil (TMI) no Brasil em 2018 foi de 13,4/1.000 nascidos vivos, seis vezes maior que a do Japão (2/1.000 nascidos vivos), uma das menores do mundo e maior que a de outros países em desenvolvimento, como a Argentina (9/1.000 nascidos vivos) ${ }^{1}$. Diferenças semelhantes também podem ser identificadas dentro do Brasil, em 2017, onde as regiões Norte e Nordeste obtiveram as maiores TMI - 17,3 e 15,8/1.000 nascidos vivos respectivamente, enquanto as regiões Sudeste e Sul apresentaram as menores, respectivamente 11,7 e 10,1/1.000 nascidos vivos. A região Centro-Oeste, por sua vez, apresentou TMI de 13/1.000 nascidos vivos $^{2}$.

A meta estabelecida frente às Nações Unidas era a redução de $66 \%$ na mortalidade materna no mundo entre 1990 e 2015 , porém, o resultado foi de $43 \%$. Cerca de $99 \%$ dos óbitos maternos ocorrem em países em desenvolvimento, cuja Razão de Mortalidade Materna (RMM) é de 239/100.000 nascidos vivos, elevada quando comparada a de países desenvolvidos $\quad(12 / 100.000 \quad$ nascidos vivos) ${ }^{3}$. A RMM no Brasil aumentou de 62 para 64/100.000 nascidos vivos entre 2015 e 2017, com diferença crescente entre as regiões brasileiras: $\mathrm{Sul}-38,5$, Centro-Oeste - 56,9, Sudeste $-62,3$, Nordeste $-73,2$ e Norte $-88,9 / 100.000$ nascidos vivos ${ }^{2}$.

A TMI é definida pelo número de crianças que morrem antes de completar um ano de idade para cada 1.000 nascidos vivos $^{4}$. Essa taxa apresenta três componentes conforme período de ocorrência do óbito: mortalidade neonatal precoce diz (óbitos ocorridos de 0 até 6 dias completos), mortalidade neonatal tardia (óbitos ocorridos de 7 a 27 dias completos) e mortalidade pós-neonatal (óbitos ocorridos de 28 a 364 dias de vida completos) ${ }^{5}$.

A morte materna diz respeito ao óbito da mulher ocorrido durante a gestação ou dentro de um período de 42 dias após a gravidez, relacionado ou agravado pela gravidez, excluindo-se causas acidentais ${ }^{6}$. É considerada direta quando ocorre por complicações obstétricas durante a gravidez, parto ou puerpério, por omissões, intervenções ou tratamento incorreto. E indireta quando por doenças prévias à gestação, agravadas por essa ou desenvolvidas nesse período, porém sem causa gestacional ${ }^{7}$. A RMM se dá pelo número de óbitos maternos por $100 \mathrm{mil}$ nascidos vivos ${ }^{5}$.

A TMI e a RMM são indicadores de saúde utilizados para analisar variações populacionais, temporais e geográficas, avaliar os níveis de saúde e desenvolvimento socioeconômico e servir de base para planejamento de políticas voltadas para a saúde, como atenção ao prénatal, parto e puerpério ${ }^{4}$. Por meio delas é possível identificar situações de iniquidades em saúde uma vez que piores desfechos em saúde estão relacionados às baixas condições socioeconômicas ${ }^{3,5}$.

$\begin{array}{ccr}\text { Além } & \text { das } & \text { condições } \\ \text { conômicas, } & \text { existem } & \text { outras }\end{array}$


influências na saúde. Essas influências e a relação entre elas pode ser demonstrada pelo modelo dos Determinantes Sociais da Saúde (DSS) proposto por Dahlgren e Whitehead $^{8}$ (1991). Tal modelo é representado por cinco camadas concêntricas, em que as mais externas influenciam e modulam as mais internas. Ainda, por meio desse modelo é possível inferir os diferentes graus de governabilidade do indivíduo sobre sua saúde e o nível de intervenção necessária por parte dos gestores.

$\mathrm{Na}$ camada mais externa estão as condições socioeconômicas, culturais e ambientais gerais que ao sofrerem interferências estruturais podem reduzir iniquidades em saúde, demonstrando sua influência sobre as outras (determinantes socioeconômicos e ambientais gerais). A próxima camada retrata o modo $\mathrm{e}$ as condições nas quais os indivíduos vivem e trabalham (por exemplo: acesso a serviços de saúde, alimentos saudáveis, educação, emprego), demonstrando que pessoas em desvantagem social são mais vulneráveis aos riscos em saúde (determinantes de condições de vida e trabalho). A terceira camada destaca a influência do apoio social de familiares, amigos ou pessoas próximas na saúde do indivíduo (determinantes de influência social). A seguir, está a camada que retrata o comportamento e estilo de vida individual, na qual as escolhas pessoais são importantes

(determinantes comportamentais), mas que claramente sofrem influências das mais externas, visto que essas escolhas dependem também de acesso à informação, pressão de amigos e familiares, acesso a alimentos e ambientes saudáveis, entre outros. Na camada mais interna estão características mais individuais como idade, sexo e condições genéticas (determinantes individuais), que possuem pouca possibilidade de mudança e são influenciadas pelas camadas mais externas.

Considerando a importância das TMI e RMM, os resultados obtidos nacionalmente e o modelo dos DSS que permite identificar as influências nos desfechos e respectivos níveis de governabilidade do gestor, esta revisão integrativa tem por objetivo compreender variáveis associadas à mortalidade materna e infantil que possam direcionar os gestores no estabelecimento de ações para o enfrentamento dessas mortalidades.

\section{Métodos}

Esta revisão foi adaptada do método Preferred Reporting Items for Systematic reviews and Meta-Analyse (PRISMA) ${ }^{9} \mathrm{e}$ envolveu as etapas de identificação, seleção, elegibilidade e inclusão. Para a identificação dos artigos, realizou-se a busca em agosto de 2018 nas bases de dados Literatura Latino-Americana e do Caribe em Ciência da Saúde (LILACS), Medical Literature Analysis and Retrieval System Online (MEDLINE) e Banco de Dados em Enfermagem (BDENF). Foram utilizados os descritores: "mortalidade infantil" AND "fatores de risco" OR "fatores de proteção" OR "fatores socioeconômicos" e "mortalidade materna" AND "fatores de risco" OR "fatores de proteção" OR "fatores socioeconômicos". Os critérios utilizados para a busca foram: publicações somente do tipo artigo original, publicados entre 1999 e 2018, com disponibilidade de texto completo, estudos feitos no Brasil e idioma português.

Após a exclusão dos artigos duplicados, duas pesquisadoras, separadamente, indicaram os artigos a serem selecionados. Para tanto realizou-se leitura do título e resumo com o critério de seleção: qualquer situação relacionada à mortalidade materna e/ou mortalidade infantil e/ou mortalidade até dois anos de idade. Para avaliar a concordância entre as pesquisadoras foi utilizado o teste de Kappa, o qual é considerado uma estatística robusta para verificar a confiabilidade entre avaliadores ${ }^{10}$. Os pesquisadores foram treinados, sendo realizadas calibrações até atingir-se um nível de concordância 
substancial ${ }^{11}$. Nos casos de discordância de indicação de seleção, os artigos foram analisados por uma terceira pesquisadora.

Os artigos selecionados foram avaliados pela leitura na íntegra. O critério de inclusão foi o uso de teste estatístico para verificação da significância da associação encontrada entre variável e desfecho estudado. Dos artigos incluídos, foram extraídas as seguintes características: título e autor para identificação do artigo, problema de pesquisa para apontar a mortalidade estudada, base de dados para reconhecimento de possíveis vieses e identificação das bases mais utilizadas, e variáveis com associação significativa à mortalidade.

Agrupamentos foram realizados em relação às fontes de $\operatorname{dados}^{12}$ : secretarias municipais e estaduais de saúde foram agrupadas como secretarias de saúde; cartórios, hospitais e unidades básicas de saúde foram agrupadas como registros oficiais; cemitérios, igrejas, delegacias, funerárias, farmácias, líderes comunitários e curandeiras como registros não oficiais.

Algumas variáveis foram agrupadas a fim de facilitar a análise dos dados: grupo afecções do recém-nascido - oligodrâmnio, hiperglicemia no recém-nascido, hipoxemia no recém-nascido, sepse neonatal e síndrome do desconforto respiratório; grupo local do nascimento - tipo de hospital, nascimento em pronto-socorro e complexidade do hospital de nascimento; grupo morbidade materna - distúrbio psiquiátrico materno, endometrite, hemorragia vaginal, agressão física em gestantes, pré-eclâmpsia e hipertensão arterial materna; grupo intercorrências da gestação - descolamento prematuro de placenta, sofrimento fetal e infecções urinárias e grupo renda - PIB per capita, razão entre a renda média dos $10 \%$ mais ricos e a dos 40\% mais pobres, concentração de renda, renda per capita e número de indigentes.

Para a análise, as variáveis foram aproximadas das camadas dos DSS, no intuito de agrupá-las para inferir o nível de governabilidade do gestor ${ }^{8}$. As camadas foram nominadas da mais interna para a mais externa: determinantes individuais, determinantes comportamentais, determinantes de influência social, determinantes de condições de vida e trabalho e determinantes socioeconômicos e ambientais gerais.

\section{Resultados}

Foram encontrados nas bases de dados MEDLINE, LILACS e BDENF, 204, 305 e 13 artigos respectivamente, totalizando 522. Desses, 304 foram excluídos por estarem duplicados e 218 avaliados por título e resumo para a seleção. O valor de Kappa obtido entre os pesquisadores para a seleção dos artigos foi de 0,793 , classificado como concordância substancial $^{11}$. Vinte e três artigos foram avaliados por uma terceira pesquisadora devido seleção discordante. Cento e quatorze artigos foram eleitos para avaliação por leitura na íntegra e no final, 74 foram incluídos na revisão (Figura 1). 
Figura 1 - Resultados de cada etapa da revisão adaptado do PRISMA

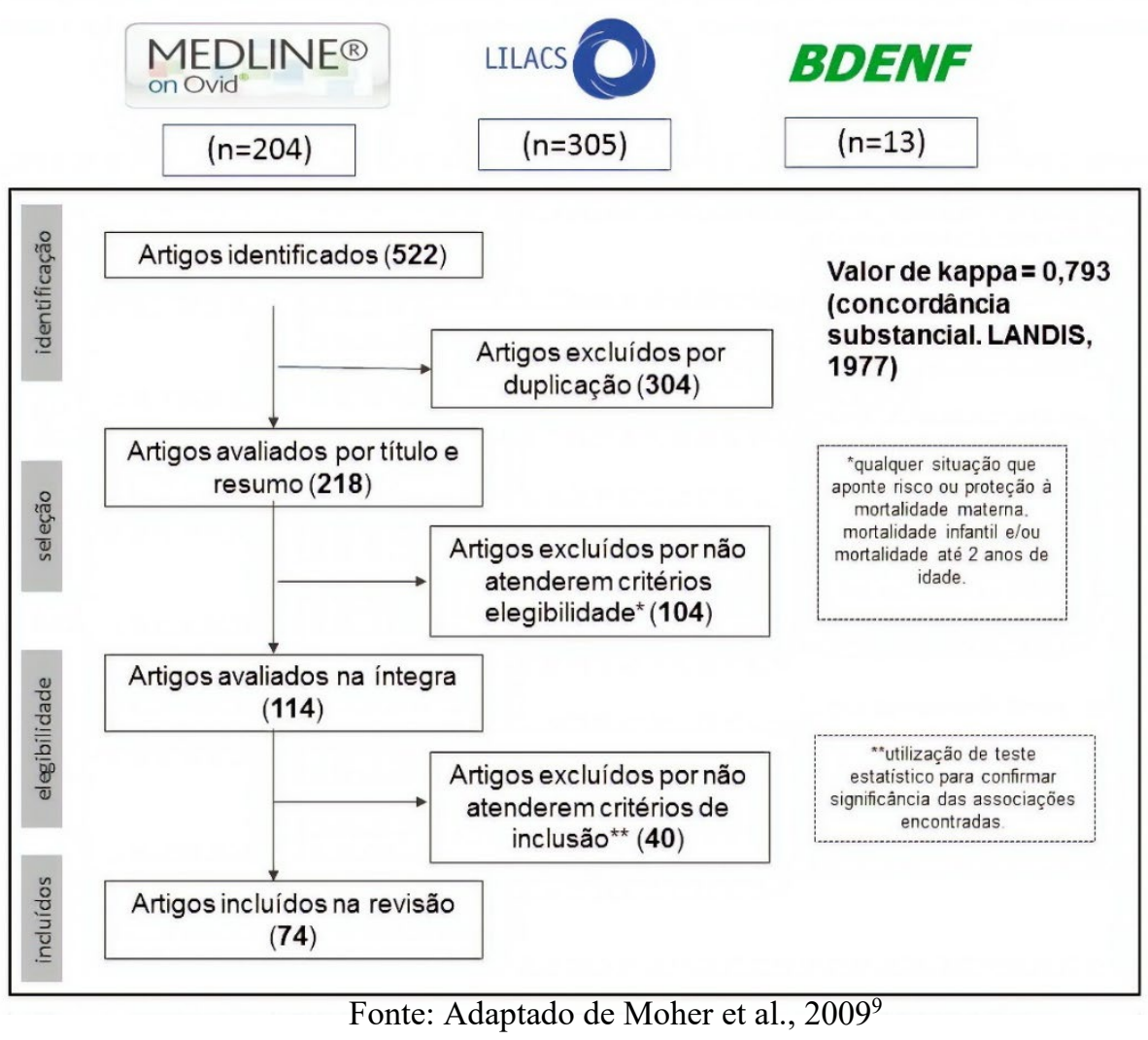

Em relação ao problema de pesquisa, 69 artigos abordaram a mortalidade infantil e desses, quase metade (31) versavam sobre mortalidade neonatal. A mortalidade materna foi estudada em seis $\operatorname{artigos}^{13-18}$, um deles associado à mortalidade infantil ${ }^{14}$. A mortalidade até dois anos foi contemplada em dois artigos, que tratava também da mortalidade infanti1 $^{19,20}$.

Sobre as fontes de dados utilizadas, destaca-se o SIM (Sistema de Informações sobre Mortalidade) e o SINASC (Sistema de Informações sobre nascidos vivos) em 40 artigos, assim como o uso de questionários em 16 e consulta a prontuários hospitalares em 12 artigos. Outras fontes utilizadas foram Instituto Brasileiro de Geografia e
Estatística (IBGE), Atlas do desenvolvimento humano no Brasil, laudos de autópsia, Fundação Nacional de Saúde e documento de alta hospitalar.

A camada dos determinantes individuais, com 27 variáveis aproximadas, foi a segunda camada mais associada às mortalidades (50 artigos), com destaque para peso ao nascer e prematuridade (30 artigos) e idade materna (14 artigos) (Tabela 1). À camada dos determinantes comportamentais foi aproximada uma variável, tabagismo na gestação, presente em um artigo (Tabela 2). A camada dos determinantes de influência social totalizou três variáveis, estado civil materno, apoio paterno na gestação e coabitação dos pais (Tabela 3). 
Tabela 1 - Variáveis aproximadas à camada dos determinantes individuais e respectivos artigos*

\begin{tabular}{|c|c|c|}
\hline variáveis & $n$ & artigo (conforme identificação nas referências) \\
\hline Peso ao nascer & 30 & $\begin{array}{c}19,22,29,32,33,34,37,40,41,42,43,44,45,46,47, \\
48,49,50,51,52,56,58,59,67,68,70,71,73,78,100\end{array}$ \\
\hline Prematuridade & 28 & $\begin{array}{l}12,22,32,33,34,35,36,37,40,41,42,43,44,46 \\
47,49,50,51,54,58,60,61,67,68,70,71,73,78\end{array}$ \\
\hline Idade materna & 14 & $16,17,22,29,30,31,33,34,35,37,47,66,70,78$ \\
\hline Tipo de parto & 13 & $16,29,30,32,33,35,36,43,54,59,66,68,71$ \\
\hline Morbidade materna & 12 & $29,36,42,52,59,60,66,67,70,72,77,80$ \\
\hline Tipo de gestação & 7 & $22,33,43,46,47,51,54$ \\
\hline Índice de Apgar no $1^{\circ}$ e $5^{\circ}$ minutos & 7 & $22,32,37,50,58,73,78$ \\
\hline Sexo & 7 & $32,33,42,44,51,52,70$ \\
\hline Cor materna & 6 & $17,19,44,47,70,78$ \\
\hline Afecções do recém-nascido & 5 & $42,59,60,61,81$ \\
\hline Número de gestações anteriores & 1 & 70 \\
\hline Número de filhos vivos & 1 & 32 \\
\hline Idade dos recém-nascidos internados em UTI & 1 & 20 \\
\hline Estatura para idade & 1 & 19 \\
\hline Peso para estatura & 1 & 19 \\
\hline História de aborto provocado & 1 & 29 \\
\hline Doenças infecciosas e parasitárias associadas à criança & 1 & 99 \\
\hline Apresentação pélvica & 1 & 51 \\
\hline Intercorrências no parto & 1 & 67 \\
\hline Nascimento pós-termo & 1 & 47 \\
\hline Índice de Apgar no $1^{\circ}$ minuto & 1 & 49 \\
\hline
\end{tabular}

*nota: um mesmo artigo pode ter apresentado mais de uma variável

Tabela 2 - Variáveis aproximadas à camada dos determinantes comportamentais e respectivos artigos*

\begin{tabular}{|c|c|c|}
\hline Variáveis & $N$ & artigo (conforme identificação nas referências) \\
\hline Tabagismo na gestação & 1 & 62 \\
\hline \multicolumn{3}{|c|}{$\begin{array}{c}\text { *nota: um mesmo artigo pode ter apresentado mais de uma variável } \\
\text { Tabela } 3 \text { - Variáveis aproximadas à camada dos determinantes de influência social e respectivos } \\
\text { artigos* }\end{array}$} \\
\hline Variáveis & $N$ & artigo (conforme identificação nas referências) \\
\hline Estado civil materno & 6 & $51,66,67,68,69,78$ \\
\hline Apoio paterno na gestação & 2 & 17,70 \\
\hline Coabitação dos pais & 1 & 32 \\
\hline
\end{tabular}

*nota: um mesmo artigo pode ter apresentado mais de uma variável

A camada dos determinantes de condições de vida e trabalho foi a que mais apareceu associada à mortalidade (56 artigos), bem como foi a que apresentou a maior diversidade de variáveis (36), com destaque para assistência pré-natal (21 artigos) e escolaridade materna (18 artigos) (Tabela 4). Por fim, à camada dos determinantes gerais foram aproximadas 19 variáveis, presentes em 21 artigos (Tabela 5), com o predomínio de saneamento básico (11 artigos) e renda (6 artigos). 
Tabela 4 - Variáveis aproximadas à camada dos determinantes de condições de vida e trabalho e respectivos artigos*

\begin{tabular}{|c|c|c|}
\hline Variáveis & $N$ & artigo (conforme identificação nas referências) \\
\hline Assistência pré-natal & 21 & $\begin{array}{c}16,22,29,32,42,44,46,51,56,66, \\
67,70,72,73,74,75,76,77,78,80,81\end{array}$ \\
\hline Escolaridade materna & 18 & $\begin{array}{c}16,17,22,33,34,35,43,45,47 \\
51,62,68,69,71,75,78,100,104\end{array}$ \\
\hline Local do nascimento & 13 & $16,22,32,34,42,46,48,50,51,60,71,74,88$ \\
\hline Residência & 7 & $12,29,30,51,75,88,94$ \\
\hline Escolaridade & 6 & $67,94,96,98,102,107$ \\
\hline Peregrinação para o parto & 5 & $18,44,51,87,88$ \\
\hline Renda familiar & 4 & $30,72,98,100$ \\
\hline Uso de corticoide antenatal & 4 & $52,59,61,81$ \\
\hline Tipo de trabalho materno & 3 & $46,66,69$ \\
\hline Condição socioeconômica materna & 2 & 19,45 \\
\hline Número de cômodos no domicílio & 2 & 67,98 \\
\hline Ventilação mecânica no recém-nascido & 2 & 51,52 \\
\hline Utilização de partograma & 2 & 42,51 \\
\hline Leitos de UTI neonatal & 2 & 53,88 \\
\hline Médicos por mil habitantes & 2 & 100,108 \\
\hline Cobertura do PACS/PSF & 2 & 100,109 \\
\hline Leitos obstétricos & 2 & 35,94 \\
\hline Número de eletrodomésticos no domicílio & 1 & 69 \\
\hline Tipo de moradia & 1 & 67 \\
\hline Meio de transporte utilizado para assistência ao parto & 1 & 67 \\
\hline Vínculo à maternidade durante o pré-natal & 1 & 76 \\
\hline Trabalho familiar na agricultura & 1 & 76 \\
\hline Consultas de puericultura & 1 & 100 \\
\hline Período de internação do recém-nascido em UTI & 1 & 20 \\
\hline Tempo de internação da gestante para o parto & 1 & 44 \\
\hline Nutrição parenteral no recém-nascido & 1 & 52 \\
\hline Uso de surfactante & 1 & 59 \\
\hline Avaliações fetais durante o trabalho de parto & 1 & 42 \\
\hline Escore de CRIB & 1 & 52 \\
\hline Critérios laboratoriais de near miss materno & 1 & 36 \\
\hline Presença ou ausência de salas de parto & 1 & 100 \\
\hline Cobertura vacinal & 1 & 100 \\
\hline Local do óbito infantil & 1 & 33 \\
\hline Número de centros e postos de saúde & 1 & 94 \\
\hline Uso de tubo endotraqueal no recém-nascido & 1 & 59 \\
\hline Internação em berçário & 1 & 29 \\
\hline
\end{tabular}

*nota: um mesmo artigo pode ter apresentado mais de uma variável

Tabela 5 - Variáveis aproximadas à camada dos determinantes gerais e respectivos artigos*

\begin{tabular}{llc}
\hline \multicolumn{1}{c}{ Variáveis } & $N$ & artigo (conforme identificação nas referências) \\
\hline \hline Saneamento básico & 11 & $93,94,96,97,98,100,101,102,104,107,108$ \\
Renda & 6 & $94,96,100,107,108,109$ \\
Região geográfica & 4 & $43,90,91,92$ \\
Taxa de fecundidade & 3 & $96,98,103$ \\
Taxa de pobreza & 3 & $19,93,98$ \\
Desnutrição infantil & 3 & $20,98,100$ \\
Taxa de urbanização & 2 & 98,100 \\
Investimentos em saúde & 2 & 107,108 \\
Esperança de vida ao nascer & 1 & 97 \\
Fornecimento de auxílio governamental & 1 & 12 \\
Turbidez da água & 1 & 102 \\
Crianças de 10 a 14 anos que trabalham & 1 & 98 \\
Índice de envelhecimento & 1 & 98 \\
Despesa pessoal em relação à despesa total em saúde & 1 & 98
\end{tabular}




\begin{tabular}{llc}
\hline \multicolumn{1}{c}{ Variáveis } & $N$ & artigo (conforme identificação nas referências) \\
\hline \hline Proporção do valor da produção vegetal em relação ao & 1 & 98 \\
total do estado & & 103 \\
Taxa de natalidade & 1 & 108 \\
Índice de Gini & 1 & 108 \\
Índice de desenvolvimento humano municipal & 1 & 13 \\
Áreas de exclusão social & 1 & \\
\hline
\end{tabular}

*nota: um mesmo artigo pode ter apresentado mais de uma variável

\section{Discussão}

A mortalidade infantil foi objeto de estudo da maioria dos artigos, com destaque para o componente neonatal. Devido à instituição de políticas globais voltadas para a diminuição da mortalidade infantil, houve um decréscimo do componente pósneonatal $^{21}$, que poderia explicar o menor número, nesta revisão, de estudos com foco nesse período. A redução desse componente colaborou para o aumento do componente neonatal no Brasil a partir da década de $90^{21}$, o que reforça a importância de estudos desse período uma vez que é o principal componente da mortalidade infantil ${ }^{22}$.

Foram encontrados poucos artigos cujo problema estudado foi a mortalidade materna, o que prejudica o aprofundamento na investigação para fatores de $\operatorname{risco}^{23}$. A maioria das mortes maternas é evitável e do tipo obstétrica, resultante de déficits na atenção à saúde da gestante desde a gestação até o puerpério ${ }^{13}$. Uma das metas preconizadas pelos Objetivos de Desenvolvimento do Milênio (ODM) era a redução da mortalidade infantil em $75 \%$ até 2015, a qual não foi alcançada no Brasil. A nova meta brasileira diante dos Objetivos de Desenvolvimento Sustentável (ODS) é de 30 mortes maternas a cada 100.000 nascidos vivos até 2030, o que contrasta com uma RMM de 64 em $2017^{24}$.

O SIM e o SINASC foram as fontes de dados mais utilizadas. São sistemas de informação em saúde (SIS) que fornecem dados sobre as mortes e nascimentos registrados no país. Seus documentos de registros são, respectivamente, a declaração de óbito (DO) e a declaração de nascido vivo $(\mathrm{DNV})^{25,26}$. Apesar da incompletude do preenchimento de tais documentos ${ }^{27}$ e da limitação na coleta de dados socioeconômicos e ambientais gerais ${ }^{28}$, o uso desses SIS como fontes de dados mais utilizadas se justifica por serem de abrangência nacional ${ }^{25,26}$.

A camada dos determinantes individuais foi a segunda mais associada às mortalidades estudadas. Apesar de ser considerada a camada com pouca possibilidade de mudanças, as características individuais são úteis para a identificação de grupos mais vulneráveis, propiciando o direcionamento das ações ${ }^{8}$. Dessa camada, a idade materna foi uma das que mais apareceram associadas à mortalidade. Gravidez na adolescência foi encontrada como uma variável de risco ${ }^{29-33}$ ao passo que idade materna maior ou igual a 35 anos foi considerada risco em outros estudos $^{34,35}$ e sem significância estatística em outro ${ }^{36}$. A influência entre as camadas do modelo dos DSS é exemplificada pela correlação da idade jovem da mãe às condições de vida, principalmente baixa inserção social, com impactos no comportamento reprodutivo e morbimortalidade das crianças ${ }^{37}$.

Ainda associado às características individuais da mãe, a infecção materna pelo vírus da dengue durante a gestação foi considerada fator de risco tanto para óbitos maternos, neonatais e infanti1 ${ }^{14}$ como apenas para óbito materno ${ }^{15}$. A infecção durante a gestação triplica o risco de óbito materno, e em casos de dengue grave o risco aumenta cerca de 27 vezes $^{38}$. Desse modo, verifica-se a influência da camada de condições de vida e trabalho do modelo dos DSS na individual à medida que os gestores propõem intervenções em relação à condição que os indivíduos vivem ${ }^{8}$, como a ampliação de ações coletivas de prevenção 
no combate à dengue e outras arboviroses, especialmente na população gestante a qual é considerada grupo vulnerável para risco de agravamento e morte por dengue ${ }^{39}$.

$\mathrm{O}$ baixo peso ao nascer foi encontrado como fator de risco ${ }^{19,34,40-47}$ enquanto outro estudo encontrou associação com a mortalidade somente o muito baixo peso $^{48}$ ou o extremo baixo peso ao nascer $^{29,49}$ O baixo peso ${ }^{29,50,51}$ e o muito baixo peso ao nascer ${ }^{52}$ são considerados uns dos principais fatores associados à mortalidade neonatal. Em contrapartida, alguns estudos não encontraram significância estatística na associação do peso ao nascer à mortalidade ${ }^{12,53,54}$. A assistência ao pré-natal de baixo risco monitora na gestante possíveis causas de diminuição do peso ao nascer, como violência, anemias, Doenças Sexualmente Transmissíveis (DST's), Infecções do Trato Urinário (ITU's) e comorbidades prévias como asma ${ }^{55}$. Dessa maneira, percebe-se, novamente, a influência das camadas mais externas do modelo dos DSS nas camadas mais internas ${ }^{8}$, em que a assistência ao prénatal (camada dos determinantes de condições de vida e trabalho) reflete no peso ao nascer (camada dos determinantes individuais).

Outra influência de camada mais externa na camada individual pode ser exemplificada pela baixa qualidade do atendimento ao neonato na sala de parto, e pode resultar em um baixo valor de Apgar $^{32,56}$. O índice de Apgar se refere à avaliação clínica do recém-nascido nos $1^{\circ} \mathrm{e}$ $5^{\circ}$ minutos de vida ${ }^{57}$, e quando menor que 7 , no $1^{\circ}$ e $5^{\circ}$ minutos, pode indicar asfixia e por isso ser previsor de risco de morte ${ }^{50}$. Encontrou-se como fator de risco quando Apgar menor do que 7 no $1^{\circ}$ minuto $^{37,58}$ e $5^{\circ}$ minuto $^{37,40,41,50,58-60}$, porém um dos estudos considerou risco quando menor ou igual a 3 no $5^{\circ}$ minuto $^{61}$.

Com relação à camada de determinantes comportamentais, o tabagismo na gestação apresentou-se como variável de risco associada à mortalidade infanti1 ${ }^{62}$. Intervenções nessa camada requerem, dentre outras, ações de educação em saúde no intuito de estimular a mudança de comportamento ${ }^{8}$. As campanhas antitabagismo são amplamente divulgadas na mídia e a televisão parece ser a mídia mais adequada para o fornecimento de materiais educativos a gestantes. Apesar das campanhas, permanece baixo o nível de conhecimento das gestantes sobre as consequências do uso do tabaco ${ }^{63}$.

O suporte social no período de gestação reduz comportamentos de risco e a chance de as crianças não terem cuidados adequados no futuro ${ }^{64,65}$. Desse modo, é importante que os gestores considerem ações que favoreçam a criação e fortalecimento de redes de apoio e autocuidado $^{8}$. O fato de a mulher não ter companheiro durante a gestação é um fator de risco para mortalidade infantil ${ }^{66-69}$, assim como o apoio paterno é fator de proteção ${ }^{70}$.

A camada dos determinantes intermediários foi a mais associada às mortalidades, além de contemplar a maior diversidade de variáveis. Dentre elas destaca-se a escolaridade materna, a qual se associou às mortalidades infantil e materna. Um tempo de escolaridade menor que quatro anos é considerado risco para mortalidade infantil ${ }^{16,34}$, assim como ausência de instrução materna ${ }^{47,71}$, enquanto a partir dos 8 anos de escolaridade as RMM se aproximaram do preconizado pela $\mathrm{OMS}^{17}$.

$\mathrm{O}$ acesso a serviços de saúde e assistência ao pré-natal podem ser inferidos pelo número de consultas de pré-natal relacionadas à mortalidade infantil quando o número de consultas for inferior a seis ${ }^{72,73}$ ou quatro para crianças que nasceram em domícilio $^{74}$, e na sua ausência ${ }^{22,29,75-77}$. O acompanhamento pré-natal de qualidade permite identificar e intervir precocemente em potenciais situações de risco que possam causar danos à saúde materno-infanti1 ${ }^{78}$, assim como vem se mostrando importante para a prevenção de morbimortalidade infantil $^{55,79,80}$ e diminuição de mortalidade de recém-nascidos prematuros ${ }^{81}$. 
Ainda relacionada à atenção à saúde materno-infantil, a criação da Rede Cegonha ocorreu em um contexto onde, apesar da ampliação na cobertura do acompanhamento pré-natal, os indicadores de mortalidade não estavam adequados e importantes causas de morbimortalidade perinatal e materna como sífilis congênita e hipertensão arterial mantinham suas taxas de incidência elevada ${ }^{55}$. De 2015 para 2016 o número de casos de sífilis em gestantes foi de 33.365 para 37.436 , e os casos de sífilis congênita, de 19.288 para $20.474^{82,83}$. A sífilis congênita é resultado do manejo inadequado de sífilis em gestantes e acarreta alta morbimortalidade para os conceptos, sendo considerada um problema de saúde pública ${ }^{84}$. Somado a isso, o não atendimento de padrões estabelecidos em protocolos nacionais e o início tardio de pré-natal são outros indícios de que apesar da alta cobertura da Atenção Pré-Natal no âmbito da Atenção Primária à Saúde, a mesma não tem sido de qualidade ${ }^{85,86}$.

Também na camada de condições de vida e trabalho, o tempo prolongado para se chegar ao local do parto se mostrou como fator de risco para mortalidade infanti1 ${ }^{18,44,51,87,88}$. Meios móveis e acessos rodoviários adequados são fatores que diminuem o tempo de acesso ao local do parto e assim contribuem para redução de óbitos infantis por causas evitáveis ${ }^{89}$.

A camada mais externa representa grande influência sobre todas as outras e a governabilidade das ações realizadas nesse nível é praticamente dos gestores, pois envolve o estabelecimento de políticas públicas além da saúde, como as voltadas para redução da pobreza ${ }^{8}$. Em regiões mais afastadas de capitais e em áreas consideradas periféricas, principalmente naquelas com condições socioeconômicas desfavoráveis, há aumento do risco de mortalidade neonatal ${ }^{90-92}$. O nascimento em regiões com altas taxas de pobreza aumenta a chance de morte neonatal ${ }^{93}$. Em uma cidade da região Nordeste, onde a taxa de mortalidade infantil é considerada alta $(15,8 / 1.000 \text { nascidos vivos })^{2}$, a mortalidade aumenta, entre outros fatores, em populações menos favorecidas situadas em zonas mais periféricas ${ }^{94}$. Os óbitos maternos são mais frequentes em locais com condições socioeconômicas baixas e em áreas rurais ${ }^{95}$.

$\mathrm{O}$ acesso à água encanada promove proteção ao desfecho de morte infanti1 ${ }^{93,94,96-98}$. Em estratos de maior carência social em que os domicílios não possuem abastecimento de água, coleta de lixo e esgotamento sanitário ligado à rede central, há maior risco de doenças infecciosas e parasitárias e mortalidade infantil $^{99-103}$. Em uma análise da evolução da mortalidade infantil na região metropolitana de São Paulo, as políticas públicas voltadas para melhoria de saneamento básico mostraram eficácia na diminuição da mortalidade pós-neonatal ${ }^{104}$.

A renda pode influenciar a saúde materno-infantil por meio do acesso à boa qualidade de alimentos, pré-natal adequado e identificação precoce de morbidades ${ }^{105}$. Iniquidades econômicas foram associadas ao nascimento de prematuros ${ }^{106}$. Maior risco de morte infantil está associado à desnutrição ${ }^{20}$, maior concentração de renda $^{107}$ e baixo índice per capita ${ }^{108}$. Associado à proteção está um maior crescimento do PIB per capita ${ }^{102,109}$. Com essas associações, evidencia-se a influência e a interação entre as camadas do modelo dos DSS e a importância de políticas públicas intersetoriais no intuito de reduzir as iniquidades em saúde, uma vez que não existe razão biológica para diferenças de expectativas de vida ${ }^{110}$.

\section{Conclusão}

A aproximação das variáveis aos DSS evidenciou a camada de condições de vida e trabalho, na qual mudanças são de governabilidade direta dos gestores. As variáveis mais associadas à mortalidade materna e infantil foram assistência prénatal, escolaridade materna, peso ao nascer, idade materna, saneamento básico e renda. 
Dado que esses fatores de risco estão bem estabelecidos e as TMI e RMM continuam aquém do esperado, as estratégias utilizadas não estão se mostrando suficientes.

\section{Agradecimentos}

Agradecemos, pelo financiamento, ao Conselho Nacional de Desenvolvimento Científico e Tecnológico - $\mathrm{CNPq}$, modalidade Iniciação Científica (IC) e ao Programa de Suporte à Pós-graduação de Instituições Comunitárias de Educação Superior - PROSUC/CAPES, modalidade taxa, para sua realização.

\section{REFERÊNCIAS}

1. UNICEF. Levels and Trends in Child Mortality Report 2019. [Acesso em 20 de abril de 2020]. Disponível em: unicef.org/media/60561/file/UM-IGME-child-mortality-report2019.pdf

2. Brasil. Ministério da Saúde. Departamento de Informação e Análise Epidemiológica. Indicadores que utilizam a metodologia do Busca Ativa. Brasília, 2019. [Acesso em 26 de junho de 2019]. Disponível em: http://svs.aids.gov.br/dantps/acesso-a-informacao/acoese-programas/busca-ativa/indicadores-de-saude/mortalidade/

3. OPAS Brasil, Folha informativa - Mortalidade materna, 2018. [Acesso em 24 junho de 2019].

Disponível

em: https://www.paho.org/bra.../index.php?option=com_content\&view=article\&id=5741:folh a-informativa-mortalidade-materna\&Itemid $=820$

4. Brasil. Ministério da Saúde. Mortalidade Perinatal Síntese de Evidências para Políticas de Saúde. Brasília, 2012. [Acesso em 27 agosto de 2019]. Disponível em: http://bvsms.saude.gov.br/bvs/publicacoes/sintese_evidencias_mortalidade_perinatal.pdf

5. OPAS. Indicadores básicos para a saúde no Brasil: conceitos e aplicações. Brasília, 2008. [Acesso em 24 junho de 2019]. Disponível em: http://tabnet.datasus.gov.br/tabdata/livroidb/2ed/indicadores.pdf

6. Brasil. Ministério da Saúde. Estudo da Mortalidade de Mulheres de 10 a 49 anos, com Ênfase na Mortalidade Materna. Relatório Final. Brasília, 2006. [Acesso em 24 de junho de 2019]. Disponível em:

http://bvsms.saude.gov.br/bvs/publicacoes/cd11_03estudo_mortalidade_mulher.pdf

7. Brasil. Ministério da Saúde. Manual dos Comitês de Mortalidade Materña. Brasília, 2007. [Acesso em 24 de junho de 2019]. Disponível em:

$\mathrm{http} / / /$ bvsms.saude.gov.br/bvs/publicacoes/comites_mortalidade_materna_3ed.pdf

8. Dahlgren G, whitehead M. Polices and strategies to promote social equity in health. Stockholm: Institute for Future Studies; 1991.

9. Moher D, Liberati A, Tetzlaff J, Altman DG, The PRISMA Group (2009) Preferred Reporting Items for Systematic Reviews and Meta-Analyses: The PRISMA Statement. PLoS Med 6(7): e1000097.

10. Marston L. Introductory Statistics for Health and Nursing Using SPSS. Thousand Oaks, California: Sage Publications, Ltd.; 2010.

11. Landis JR, Koch GG. The measurement of observer agreement for categorical data. Biometrics, 1977; 33:59-174. 
12. Barbosa TAGS, Coelho KR, Andrade GN, Bittencourt SDA, Leal MC, Gazzinelli A. Determinantes da mortalidade infantil em municípios do Vale do Jequitinhonha, Minas Gerais, Brasil. Rev. Mineira de Enfermagem. 2014;18(4):907-914.

13. Faria DR, Sousa RC, Costa TJNM, Leite ICG. Mortalidade materna em cidade-polo de assistência na região Sudeste: tendência temporal e determinantes sociais. Rev. Med. Minas Gerais. 2012;22(1):1-128.

14. Feitoza HAC, Koifman S, Koifman RJ, Saraceni V. Os efeitos maternos, fetais e infantis decorrentes da infecção por dengue durante a gestação em Rio Branco, Acre, Brasil, 2007-2012. Cad. Saúde Pública. 2017;33(5):e00178915.

15. Mota AKM, Filho ALM, Saraceni V, Koifman S. Mortalidade materna e incidência de dengue na Região Sudeste do Brasil: estudo ecológico no período 2001-2005. Cad. Saúde Pública. 2012;28(6):1057-1066.

16. Leite RMB, Araújo TVB, Albuquerque RM, Andrade ARS, Neto PJD. Fatores de risco para mortalidade materna em área urbana do Nordeste do Brasil. Cad. Saúde Pública. 2011;27(10):1977-1985.

17. Carreno I, Bonilha ALL, Costa JSD. Perfil epidemiológico das mortes maternas ocorridas no Rio Grande do Sul, Brasil: 2004-2007. Rev. Bras. Epidemiologia. 2012;15(2):396406.

18. Troncon JK, Netto DLQ, Rehder PM, Cecatti JG, Surita FG. Mortalidade materna em um centro de referência do Sudeste Brasileiro. Rev Bras Ginecol Obstet. 2013;35(9):388393.

19. Horta BL, Gigante DP, Candiota JS, Barros FC, Victora CG. Monitorização da mortalidade na coorte de nascimentos de 1982 a 2006, Pelotas, RS. Rev Saúde Pública. 2008;42(Supl. 2):108-114.

20. Einloft PR, Garcia PC, Piva JP, Bruno F, Kipper DJ, Fiori RM. Perfil epidemiológico de dezesseis anos de uma unidade de terapia intensiva pediátrica. Rev Saúde Pública. 2002;36(6):728-733.

21. Lansky S, França E, Leal MC. Mortalidade perinatal e evitabilidade: revisão de literatura. Rev Saúde Pública. 2002;36(6):759-772.

22. Lima EFA, Sousa AI, Griep RH, Primo CC. Fatores de risco para mortalidade neonatal no município de Serra, Espírito Santo. Rev. Bras. Enferm. 2012;65(4):578-85.

23. Morse ML, Fonseca SC, Barbosa MD, Calil MB, Eyer FPC. Mortalidade materna no Brasil: o que mostra a produção científica nos últimos 30 anos?. Cad. Saúde Pública. 2011;27(4):623-638.

24. Portal ODS. Mortalidade materna cresce no Brasil. [Acesso em 27 de agosto de 2019]. Disponível em: http://portalods.com.br/noticias/mortalidade-materna-cresce-no-brasil/

25. Brasil. Ministério da Saúde. Manual de Instruções para o preenchimento da Declaração de Óbito. Séria A. Normas e Manuais Técnicos. Brasília, 2011. [Acesso em 2 de maio de 2020]. Disponível em: http://svs.aids.gov.br/download/manuais/Manual_Instr_Preench_DO_2011_jan.pdf

26. Brasil. Ministério da Saúde. Manual de Instruções para o preenchimento da Declaração de Nascido Vivo. Séria A. Normas e Manuais Técnicos. Brasília, 2011. [Acesso em 2 de maio de 2020]. Disponível em: http://portalarquivos.saude.gov.br/images/pdf/2017/junho/08/inst_dn.pdf

27. Maia LTS, Souza WV, Mendes ACG, Silva AGS. Uso do linkage para a melhoria da completude do SIM e do Sinasc nas capitais brasileiras. Rev. Saúde Pública. 2017; 51:112.

28. Soares DC, Facchini LA, Casarin ST, Thumé E. Programas de vigilância de recémnascidos de risco: experiências municipais brasileiras. Cultura de los Cuidados. 2019;23(53). 
29. Almeida MF, Alencar GP, Schoeps D, Novaes HMD, Campbell O, Rodrigues LC. Sobrevida e fatores de risco para mortalidade neonatal em uma coorte de nascidos vivos de muito baixo peso ao nascer, na Região Sul do Município de São Paulo, Brasil. Cad. Saúde Pública. 2011;27(6):1088-1098.

30. Andrade CLT, Szwarcwald CL. Análise espacial da mortalidade neonatal precoce no Município do Rio de Janeiro, 1995-1996. Cad. Saúde Pública. 2001;17(5):1199-1210.

31. Simões VMF, Silva AAM, Bettiol H, Filho FL, Tonial SR, Mochel EG. Características da gravidez na adolescência em São Luís, Maranhão. Rev Saúde Pública. 2003;37(5):559-565.

32. Ribeiro AM, Guimarães MJ, Lima MC, Sarinho SW, Coutinho SB. Fatores de risco para mortalidade neonatal em crianças com baixo peso ao nascer. Rev Saúde Pública. 2009;43(2):246-255.

33. Mombelli MA, Sass A, Molena CAF, Téston EF, Marcon SS. Fatores de risco para mortalidade infantil em municípios do Estado do Paraná, de 1997 a 2008. Rev Paul Pediatr. 2012;30(2):187-194.

34. Aquino TA, Guimarães MJB, Sarinho SW, Ferreira LOC. Fatores de risco para a mortalidade perinatal no Recife, Pernambuco, Brasil, 2003. Cad. Saúde Pública. 2007;23(12):2853-2861.

35. Gonçalves AC et al. Tendência da mortalidade neonatal na cidade de Salvador (BahiaBrasil), 1996-2012. Rev. Bras. Saude Mat. Infant. 2015;15(3):337-347.

36. Oliveira LC, Costa AAR. Óbitos fetais e neonatais entre casos de near miss materno. Rev. Assoc. Med. Bras. 2013;59(5):487-494.

37. Kropiwiec MV, Franco SC, Amaral AR. Fatores associados à mortalidade infantil em municípios com índice de desenvolvimento humano elevado. Rev Paul Pediatr. 2017;35(4):391-398.

38. Paixão ES et al. Dengue in pregnancy and maternal mortality: a cohort analysis using routine data. Scientific Reports. 2018;8(1):1-6.

39. Nascimento LB, Siqueira CM, Coelho GE, Junior JBS. Dengue em gestantes: caracterização dos casos no Brasil, 2007-2015*. Epidemiol. Serv. Saúde. 2017;26(3):433442.

40. Doldan RV, Costa JSD, Nunes MF. Fatores associados à mortalidade infantil no Município de Foz do Iguaçu, Paraná, Brasil: estudo de caso-controle. Epidemiol. Serv. Saúde. 2011;20(4):491-498.

41. Fréu CM, Mottin LM, Migott MD, Martinelli IB, Nunes ML, Geib LTC. Determinantes da mortalidade neonatal de uma coorte de nascidos vivos em Passo Fundo - RS, 20032004. Revista da AMRIGS. 2008; 52(2):97-102.

42. Lansky S, França E, César CC, Neto LCM, Leal MC. Mortes perinatais e avaliação da assistência ao parto em maternidades do Sistema Único de Saúde em Belo Horizonte, Minas Gerais, Brasil, 1999. Cad. Saúde Pública. 2006;22(1):117-130.

43. Shimakura SE, Carvalho MS, Aerts DRGC, Flores R. Distribuição espacial do risco: modelagem da mortalidade infantil em Porto Alegre, Rio grande do Sul, Brasil. Cad. Saúde Pública. 2001;17(5):1251-1261.

44. Nascimento RM, Leite AJM, Almeida NMGS, Almeida PC, Silva CF. Determinantes da mortalidade neonatal: estudo caso-controle em Fortaleza, Ceará, Brasil. Cad. Saúde Pública. 2012;28(3):559-572.

45. Hernandez AR, Silva CH, Agranonik M, Quadros FM, Goldani MZ. Análise de tendências das taxas de mortalidade e de seus fatores de risco na cidade de Porto Alegre, Rio Grande do Sul, Brasil, no período de 1996 a 2008. Cad. Saúde Pública. 2011;27(11):2188-2196. 
46. Nascimento EMR, Costa MCN, Mota ELA, Paim JS. Estudo de fatores de risco para óbitos de menores de um ano mediante compartilhamento de bancos de dados. Cad. Saúde Pública. 2008;24(11):2593-2602.

47. Teixeira GA, Costa FML, Mata MS, Carvalho JBL, Souza NL, Silva RAR. Fatores de risco para a mortalidade neonatal na primeira semana de vida. Rev. Pesquisa. Cuid. Fundam. 2016;8(1):4036-4046.

48. Pinheiro CEA, Peres MA, d'Orsi E. Aumento na sobrevida de crianças de grupos de peso baixo ao nascer em Santa Catarina. Rev Saúde Pública. 2010;44(5):776-784.

49. Oliveira TG et al. Escore de Apgar e mortalidade neonatal em um hospital localizado na zona sul do município de São Paulo. Einstein (São Paulo). 2012;10(1):22-28.

50. Ferrari RAP, Bertolozzi MR, Dalmas JC, Girotto E. Fatores determinantes da mortalidade neonatal em um município da Região Sul do Brasil. Rev Esc Enferm USP. 2013; 47(3):531-538.

51. Lansky S et al. Pesquisa Nascer no Brasil: perfil da mortalidade neonatal e avaliação da assistência à gestante e ao recém-nascido. Cad. Saúde Pública. 2014; 30Sup:S192-S207.

52. Duarte JLMB, Mendonça GAS. Fatores associados à morte neonatal em recém-nascidos de muito baixo peso em quatro maternidades no Município do Rio de Janeiro, Brasil. Cad. Saúde Pública. 2005;21(1):181-191.

53. Oliveira GS, Lima MCBM, Lyra CO, Oliveira AGRC, Ferreira MAF. Desigualdade espacial da mortalidade neonatal no Brasil: 2006 a 2010. Ciênc. Saúde Coletiva. 2013;18(8):2431-2441.

54. Sanders LSC, Pinto FJM, Medeiros CRB, Sampaio RMM, Viana RAA, Lima KJ. Mortalidade infantil: análise de fatores associados em uma capital do Nordeste Brasileiro. Cad. Saúde Colet. 2017;25(1):83-89.

55. Brasil. Ministério da saúde. Atenção ao pré-natal de baixo risco. Cadernos de Atenção Básica. Brasília, 2012. [Acesso em 24 de junho de 2019]. Disponível em: http://bvsms.saude.gov.br/bvs/publicacoes/cadernos_atencao_basica_32_prenatal.pdf

56. Paulucci RS, Nascimento LFC. Mortalidade neonatal em Taubaté: um estudo casocontrole. Rev Paul de Pediatr. 2007;25(4):358-363.

57. Apgar V. A proposal for a new method of evaluation of the newborn infant. Curr Res Anesth Analg. 1953;32(4):260-267.

58. Maran E, Uchimura TT. Mortalidade neonatal: fatores de risco em um município no sul do Brasil. Rev. Eletr. de Enfermagem. 2008;10(1):29-38.

59. Silva CF, Leite AJM, Almeida NMGS, Leon ACMP, Olofin I, Perinatal RNNS. Fatores associados ao óbito neonatal de recém-nascidos de alto risco: estudo multicêntrico em Unidades Neonatais de Alto Risco no Nordeste brasileiro. Cad. Saúde Pública. 2014;30(2):355-368.

60. Almeida MFB et al. Fatores perinatais associados ao óbito precoce em prematuros nascidos nos centros da Rede Brasileira de Pesquisas Neonatais. J Pediatr. 2008;84(4):300-307.

61. Prigenzi MLH, Trindade CEP, Rugolo LMSS, Silveira LVA. Fatores de risco associados à mortalidade de recém-nascidos de muito baixo peso na cidade de Botucatu, São Paulo, no período 1995-2000. Rev. Bras. Saúde Mater. Infant. 2008;8(1):93-101.

62. Geib LTC, Fréu CM, Brandão M, Nunes ML. Determinantes sociais e biológicos da mortalidade infantil em coorte de base populacional em Passo Fundo, Rio Grande do Sul. Ciênc. Saúde Coletiva. 2010;15(2):363-370.

63. Bertani AL, Garcia T, Tanni SE, Godoy I. Prevenção do tabagismo na gravidez: importância do conhecimento materno sobre os malefícios para a saúde e opções de tratamento disponíveis. J. Bras. Pneumologia. 2015;41(2):175-181. 
64. Cunha AJLA, Leite AJM, Almeida IS. The pediatrician's role in the first thousand days of the child: the pursuit of healthy nutrition and development. J Pediatr. 2015;91(6): S44S51.

65. Moore TG, Arefabid N, Derry A, West S. The First Thousand Days: An Evidence Paper. Murdoch Children's Research Institute: Melbourne, Centre for Community Child Health, 2017. [Acesso em 03 de junho de 2019]. Disponível em:

https://www.suicideinfo.ca/resource/the-first-thousand-days-an-evidence-paper/

66. Ribeiro FD, Ferrari RAP, Sant'Anna FL, Dalmas JC, Girotto E. Extremos de idade materna e mortalidade infantil: análise entre 2000 e 2009. Rev Paul Pediatr. 2014;32(4):381-388.

67. Schoeps D et al. Fatores de risco para mortalidade neonatal precoce. Rev Saúde Pública. 2007;41(6):1013-1022.

68. Jobim R, Aerts D. Mortalidade infantil evitável e fatores associados em Porto Alegre, Rio Grande do Sul, Brasil, 2000-2003. Cad. Saúde Pública. 2008;21(1):179-187.

69. França E, Souza JM, Guimarães MDC, Goulart EMA, Colosimo E, Antunes CMF. Associação entre fatores sócio-econômicos e mortalidade infantil por diarréia, pneumonia e desnutrição em região metropolitana do Sudeste do Brasil: um estudo caso-controle. Cad. Saúde Pública. 2001;17(6): 1437-1447.

70. Oliveira EFV, Gama SGN, Silva CMFP. Gravidez na adolescência e outros fatores de risco para mortalidade fetal e infantil no Município do Rio de Janeiro, Brasil. Cad. Saúde Pública. 2010;26(3):567-578.

71. Neto OLM, Barros MBA. Fatores de risco para mortalidade neonatal e pós-neonatal da Região Centro-Oeste do Brasil: linkage entre bancos de dados de nascidos vivos e óbitos infantis. Cad. Saúde Pública. 2000;16(2):477-485.

72. Pereira PK, Vieira CL, Santos JFC, Lima LA, Legay LF, Lovisi GM. Avaliação de desfechos perinatais/infantis em partos de pacientes com transtornos mentais maiores de um hospital psiquiátrico do Rio de Janeiro, Brasil. Cad. Saúde Pública. 2014;30(8):16541666.

73. Silva CF, Leite AJM, Almeida NMGS, Gondim RC. Fatores de risco para mortalidade infantil em município do Nordeste do Brasil: linkage entre bancos de dados de nascidos vivos e óbitos infantis - 2000 a 2002. Rev. Bras. Epidemiologia. 2006;9(1):69-80.

74. Silva ZP et al. Características dos nascidos vivos, das mães e mortalidade neonatal precoce na Região Metropolitana de São Paulo, Brasil. Cad. Saúde Pública. 2009;25(9):1981-1989.

75. Giglio MRP, Lamounier JA, Neto OLM. Via de parto e risco para mortalidade neonatal em Goiânia no ano de 2000. Rev. Saúde Pública. 2005;39(3):350-357.

76. Leal MC, Bittencourt SDA, Torres RMC, Niquini RP, Junior PRBS. Determinantes do óbito infantil no Vale do Jequitinhonha e nas regiões Norte e Nordeste do Brasil. Rev Saúde Pública. 2017; 51:12.

77. Almeida MF et al. Partos Domiciliares acidentais na região sul do Município de São Paulo. Rev Saúde Pública. 2005;39(3):366-375.

78. Maia LTS, Souza WV, Mendes ACG. Diferenciais nos fatores de risco para a mortalidade infantil em cinco cidades brasileiras: um estudo de caso-controle com base no SIM e no SINASC. Cad. Saúde Pública. 2012;28(11):2163-2176.

79. Araújo BF, Bozzetti MC, Tanaka AC. A. Mortalidade neonatal precoce no município de Caxias do Sul: um estudo de coorte. J Pediatr. 2000;76(3):200-206.

80. Viellas EF, Gama SGN, Carvalho ML, Pinto LW. Fatores associados à agressão física em gestantes e os desfechos negativos no recém-nascido. J Pediatr. 2013;89(1):83-90.

81. Araújo BF et al. Influência do local de nascimento e do transporte sobre a morbimortalidade de recém-nascidos prematuros. J Pediatr. 2011;87(3):257-262. 
82. Brasil. Ministério da saúde. Boletim Epidemiológico - Sífilis 2016. Secretária de Vigilância em Saúde. [Acesso em 24 de junho de 2019]. Disponível em: http://portalarquivos2.saude.gov.br/images/pdf/2016/outubro/31/2016_030_Sifilispublicao2.pdf

83. Brasil. Ministério da saúde. Boletim Epidemiológico - Sífilis 2017. Secretária de Vigilância em Saúde. [Acesso em 24 de junho de 2019]. Disponível em: http://portalarquivos.saude.gov.br/images/pdf/2017/novembro/13/BE-2017-038-BoletimSifilis-11-2017-publicacao-.pdf

84. Cardoso ARP, Araújo MAL, Cavalcante MS, Frota MA, Melo SP. Análise dos casos de sífilis gestacional e congênita nos anos de 2008 a 2010 em Fortaleza, Ceará, Brasil. Ciênc. saúde colet. 2018;23(2):563-574.

85. Luz LA, Aquino R, Medina MG. Avaliação da qualidade da Atenção Pré-Natal no Brasil. Saúde Debate. 2018;42(2):111-126.

86. Mendes RB et al. Avaliação da qualidade do pré-natal a partir das recomendações do programa de humanização no pré-natal e nascimento. Ciên e saúde colet. [Acesso em 29 de agosto de 2019]. Disponível:

http://www.cienciaesaudecoletiva.com.br/artigos/avaliacao-da-qualidade-do-prenatal-apartir-das-recomendacoes-do-programa-de-humanizacao-no-prenatal-enascimento/16895? id $=16895$

87. Almeida WS, Szwarcwald CL. Mortalidade infantil e acesso geográfico ao parto nos municípios brasileiros. Rev Saúde Pública. 2012;46(1):68-76.

88. Pereira APE, Gama SGN, Leal MC. Mortalidade infantil em uma amostra de nascimentos do município do Rio de Janeiro, 1999-2001: "linkage" com o Sistema de Informação de Mortalidade. Rev. Bras. Saúde Mater. Infant. 2007;7(1):83-88.

89. Zuanazzi PT, Cabral PHV, Stella MA, Moraes GI. O tempo de percurso a cidades polos regionais influencia na mortalidade em pequenos municípios gaúchos. Cad. Saúde Pública. 2017;33(12): e00187515.

90. Almeida MCS, Gomes CMS, Nascimento LFC. Análise espacial da mortalidade neonatal no estado de São Paulo, 2006-2010. Rev Paul Pediatr. 2014;32(4):374-380.

91. Nascimento LFC, Bastista GT, Dias NW, Catelani CS, Becker D, Rodrigues L. Análise espacial da mortalidade neonatal no Vale do Paraíba, 1999 a 2001. Rev Saúde Pública. 2007;41(1):94-100.

92. Neto OLM, Barros MBA, Martelli CMT, Silva SA, Cavenaghi SM, Jr JBS. Diferenças no padrão de ocorrência da mortalidade neonatal e pós-neonatal no Município de Goiânia, Brasil, 1992-1996: análise espacial para identificação das áreas de risco. Cad. Saúde Pública. 2001;17(5):1241-1250.

93. Zanini RR, Moraes AB, Giugliani ERJ, Riboldi J. Determinantes contextuais da mortalidade neonatal no Rio Grande do Sul por dois modelos de análise. Rev Saúde Pública. 2011;45(1):79-89.

94. Costa MCN, Azi PA, Paim JS, Silva LMV. Mortalidade infantil e condições de vida: a reprodução das desigualdades sociais em saúde na década de 90. Cad. Saúde Pública. 2001;17(3):555-567.

95. Passos ET, Rodrigues GMM. Taxas de mortalidade materna no Brasil. Revista Liberum Accessum. 2020;1(1).

96. Sousa TRV, Filho PAML. Análise por dados em painel do status de saúde no Nordeste Brasileiro. Rev Saúde Pública. 2008;42(5):796-804.

97. Teixeira JC, Guilhermino RL. Análise da associação entre saneamento e saúde nos estados brasileiros, empregando dados secundários do banco de dados indicadores e dados básicos para a saúde 2003 - IDB 2003. Eng. Sanit. Ambient. 2006;11(3):277-282. 
98. Filho JGB, Pontes LRFSK, Miná DL, Barreto ML. Mortalidade infantil e condições sociodemográficas no Ceará, em 1991 a 2000. Rev Saúde Pública. 2007;41(6):10231031.

99. Vilela MBR, Bonfim C, Medeiros Z. Mortalidade infantil por doenças infecciosas e parasitárias: reflexo das desigualdades sociais em um município do Nordeste do Brasil. Rev. Bras. Saúde Mater. Infant. 2008;8(4):455-461.

100. Filho JGB, Kerr LRFS, Miná DL, Barreto ML. Distribuição espacial da taxa de mortalidade infantil e principais determinantes no Ceará, Brasil, no período 2000-2002. Cad. Saúde Pública. 2007;23(5):1173-1185.

101. Bühler HF, Ignotti E, Neves SMAS, Hacon SS. Análise espacial de indicadores integrados determinantes da mortalidade por diarreia aguda em crianças menores de 1 ano em regiões geográficas. Ciênc. Saúde Coletiva. 2014;19(10):4131-4140.

102. Costa SS, Heller L, Brandão CCS, Colosimo EA. Indicadores sanitários sentinelas da mortalidade infantil: um estudo ecológico de base municipal em quatro estados brasileiros. Cad. Saúde Coletiva. 2005;13(1):131-150.

103. Costa MCN, Mota ELA, Paim JS, Silva LMV, Teixeira MG, Mendes CMC. Mortalidade infantil no Brasil em períodos recentes de crise econômica. Rev Saúde Pública. 2003;37(6):699-706.

104. Holcman MM, Latorre MRDO, Santos JLF. Evolução da mortalidade infantil na região metropolitana de São Paulo, 1980-2000. Rev Saúde Pública. 2004;38(2):180-186.

105. Blencowe $\mathrm{H}$ et al. Born Too Soon: The global epidemiology of 15 million preterm births. Reproductive Health. 2013;10(1): S2.

106. Sadovsky ADI, Matijasevich A, Santos IS, Barros FC, Miranda AE, Silveira MF. Iniquidades socioeconômicas em nascimentos prematuros em quatro estudos brasileiros de coortes de nascimento. J Pediatr. 2018;94(1):15-22.

107. Fischer TK, Lima D, Rosa R, Osório D, Boing AF. A mortalidade infantil no Brasil: série histórica entre 1994-2004 e associação com indicadores socioeconômicos em municípios de médio e grande porte. Rev. Medicina Ribeirão Preto. 2007;40(4):559-566.

108. Boing AF, Boing AC. Mortalidade infantil por causas evitáveis no Brasil: um estudo ecológico no período 2000-2002. Cad. Saúde Pública. 2008;24(2)447-455.

109. Lourenço EC et al. Variáveis de impacto na queda da mortalidade infantil no Estado de São Paulo, Brasil, no período de 1998 a 2008. Ciênc. Saúde Coletiva. 2014;19(7):20552062.

110. Marmot M. Social determinants of health inequalities. Lancet. 2005; 365:1099-1104.

\section{Como citar este artigo:}

Martins IPM, Nakamura CY, Carvalho DR. Variáveis associadas à mortalidade materna $\mathrm{e}$ infantil: uma revisão integrativa. Rev. Aten. Saúde. 2020; 18(64): 149-165. 